\title{
Hydrogen Solubility of the Molten Salt FLiNaK Mixed with Nano-Ti Powder*)
}

\author{
Juro YAGI, Akio SAGARA, Teruya TANAKA, Sadatsugu TAKAYAMA and Takeo MUROGA \\ National Institute for Fusion Science, National Institutes of Natural Sciences, Toki, Gifu 509-5292, Japan
}

(Received 30 November 2015 / Accepted 5 April 2016)

\begin{abstract}
The hydrogen-release behavior of the molten salt FLiNaK containing directly synthesized $0.1 \mathrm{wt} \%$ nanoTi powder was investigated. Hydrogen release was faster at higher temperatures, and the volume of released hydrogen from FLiNaK was significantly higher than that from pure FLiNaK. However, the ratio of released $\mathrm{H} / \mathrm{Ti}$ in FLiNaK was smaller than that in $\mu \mathrm{m}$-Ti powder.
\end{abstract}

(C) 2016 The Japan Society of Plasma Science and Nuclear Fusion Research

Keywords: molten salt, FLiNaK, enhancement of effective hydrogen solubility, metal powder, nanopowder

DOI: $10.1585 /$ pfr. 11.2405099

\section{Introduction}

The liquid blanket that transports heat and tritium generated in a fusion reactor via a lithium-containing liquid simplifies the reactor structure and is expected to achieve high energy efficiency. Molten fluoride salts such as FLiBe, FLiNaBe, and FLiNaK and liquid metals such as pure lithium and lead-lithium eutectic have been previously proposed as candidates for liquid breeding materials. Compared with liquid metals, molten salts are preferred for their low reactivity with air and water and low electric resistivity (free from MHD (magnetohydrodynamics) pressure drop in a magnetic fusion reactor). In addition, wellpurified molten salts have good compatibility with reactor structure materials. However, the major concern regarding molten salts is their low solubility of molecular hydrogen ( $T_{2}$ in a nuclear environment); this increases the risk of tritium leakage and tritium inventory in structure materials. Moreover, impurities such as water, TF, and free F get generated via neutron irradiation on lithium fluoride; this reduces compatibility of molten salts with structure materials.

The concept of mixing metal powders with molten salts was first proposed by A. Sagara [1]. Mixing metal powders that are highly soluble of hydrogen (e.g., titanium) with molten salts not only increases the effective hydrogen solubility but also traps impurities such as water and hydrogen fluoride with no MHD effects. The effectiveness of titanium powder mixed in FLiNaK was confirmed in our previous work [2], which reported that a weight ratio of $0.1 \mathrm{wt} . \%$ is sufficient to increase the hydrogen solubility of FLiNaK by several orders of magnitude and that the oxidation of the powder deactivates hydrogen absorption.

In this work, using the previously produced nano-Ti powder mixed in FLiNaK, the hydrogen release behavior

author's e-mail: yagi.juro@LHD.nifs.ac.jp

*) This article is based on the presentation at the 25th International Toki Conference (ITC25). and the effective hydrogen solubility of the powder were investigated to elucidate the effect of the powder production method.

\section{Experimental}

In this study, molten salts containing titanium powder were synthesized using the plasma-induced discharge electrolysis method (I'MSEP Co., Ltd, Japan) [3]. The electrolytic bath was filled with FLiNaK and was equipped with a titanium anode. The cathode, which is usually dipped into the molten salt, was lifted above the liquid surface to enable discharge to the molten salt. The electron injected into the molten salt reacted with the titanium ion to produce very fine titanium metal powder. The reduction procedure continued until the amount of discharged electrons reached the level that is necessary to produce $0.1 \mathrm{wt} \%$ Ti from a titanium ion.

Because itanium powder was directly reduced from titanium ions in FLiNaK, its surface was expected to be free from oxidizing contamination. The actual diameter of the titanium particle used in this work was not measured because this direct-reduction method produces typical sizes with a primary diameter of approximately $10 \mathrm{~nm}$ and an agglomerated secondary diameter of approximately $100 \mathrm{~nm}$. Pure molten salt was used as a control in this experiment and was synthesized from a special-grade fluoride powder (LiF, NaF, and $\mathrm{KF}$ ) produced by Kanto chemical Co., Ltd., Japan.

Almost $40 \mathrm{~g}$ of molten salt was contained in a quartz tube installed in a ring furnace. A gas injection tube (3.2$\mathrm{mm}$ diameter, $0.5-\mathrm{mm}$ thick) made of pure $\mathrm{Ni}$ was immersed into the liquid, and hydrogen-containing argon gas and pure argon gas were supplied for hydrogen charging and hydrogen release, respectively. The concentration of hydrogen in argon gas was monitored via a gas chromatograph (Shimadzu, GC-8A) for each 5 min or longer. The experimental setup is shown in Fig. 1. 


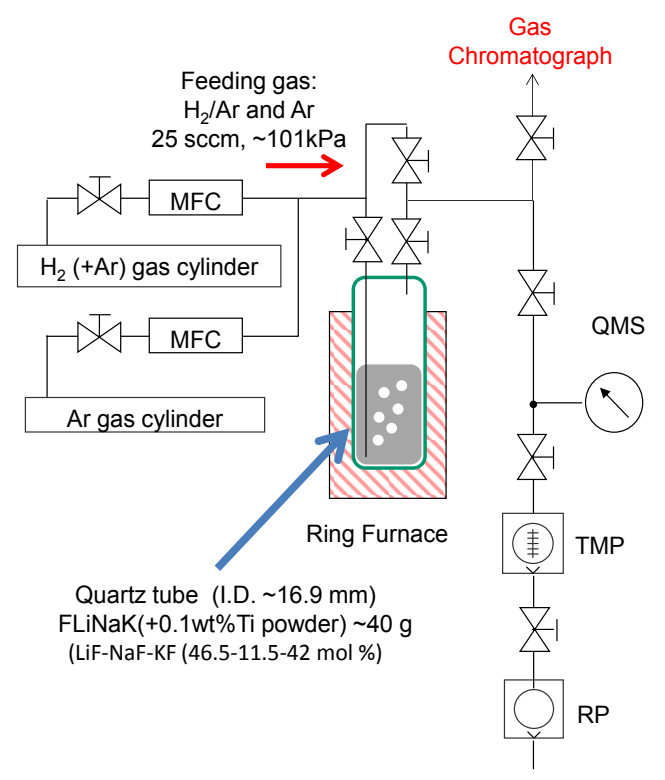

Fig. 1 Experimental setup of the hydrogen supply and extraction system.

Hydrogen supply and extraction were performed with $25 \mathrm{sccm}$ of $1.02 \% \mathrm{H}_{2} / \mathrm{Ar}$ gas and pure Ar gas, respectively. The gases were injected at a pressure slightly higher than the ambient pressure, and the hydrogen equilibrium pressure during hydrogen supply was assumed to be $1.03 \mathrm{kPa}$. Hydrogen supply was performed for more than $3 \mathrm{~h}$ followed by hydrogen extraction and replacing the gas with pure Ar. Hydrogen supply and extraction were performed at $798,853,903$, and $973 \mathrm{~K}$.

\section{Results and Discussion}

Hydrogen-release behavior of the Ti-containing FLiNaK and pure FLiNaK are shown in Fig. 2 - Fig. 5. The sum of two exponentials was used to fit the plots before $3000 \mathrm{~s}$ and more hydrogen was released from the Ticontaining FLiNaK at all temperatures because of the hydrogen release from the Ti powder.

Hydrogen-release behavior of Ti-containing FLiNaK is also plotted in Fig. 6. Faster hydrogen release from the liquid at higher temperatures is clearly observed. The difference between the hydrogen release from Ti-containing FLiNaK and from pure FLiNaK (assumed to be released from the Ti powder) is calculated and integrated from the results. The integration is performed on the fitting exponential functions, starting from $500-3000 \mathrm{~s}$ and from $500 \mathrm{~s}$ to an infinite time period. As shown in Fig. 7, the integration up to $3000 \mathrm{~s}$ is almost equal to the experimental value; however, integrating to infinity will underestimate and overestimate at $903 \mathrm{~K}$ and $973 \mathrm{~K}$, respectively. This is because of the accuracy of the fitting curve. The integrated values are shown in Fig. 7 with those acquired in our previous work [2], which was performed using $\mu \mathrm{m}$ size Ti powder charged by $5-\mathrm{kPa}_{2}$ gas. The $\mathrm{H}_{2}$ pressure in the previous work was five times higher than that in the

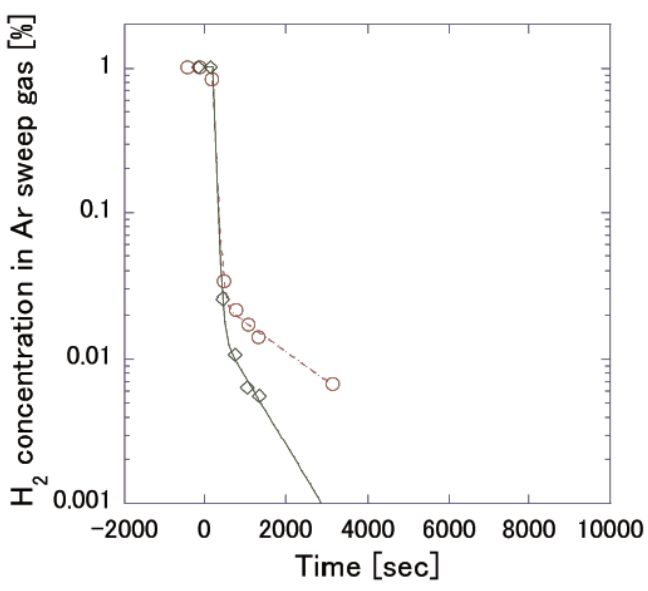

Fig. 2 Hydrogen release behavior of FLiNaK and Ti-containing FLiNaK at $798 \mathrm{~K}$. Diamond and solid line: pure FLiNaK. Circle and dashed line: Ti-mixed FLiNaK.

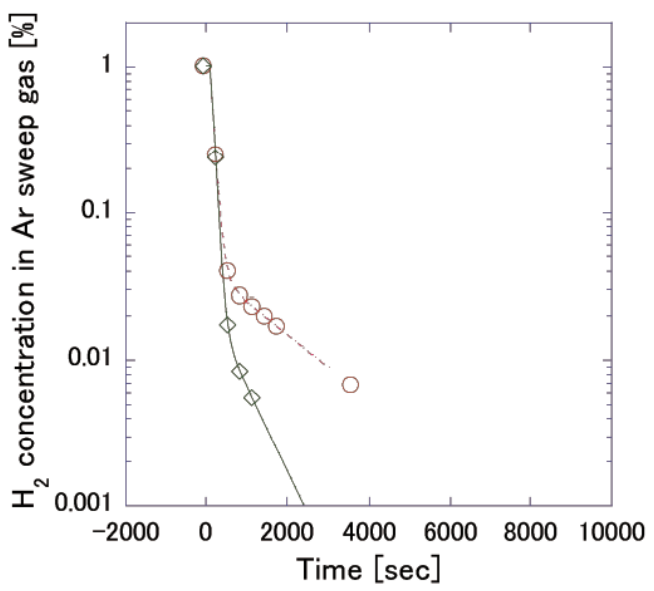

Fig. 3 Hydrogen release behavior of FLiNaK and Ti-containing FLiNaK at $853 \mathrm{~K}$. Diamond and solid line: pure FLiNaK. Circle and dashed line: Ti-mixed FLiNaK.

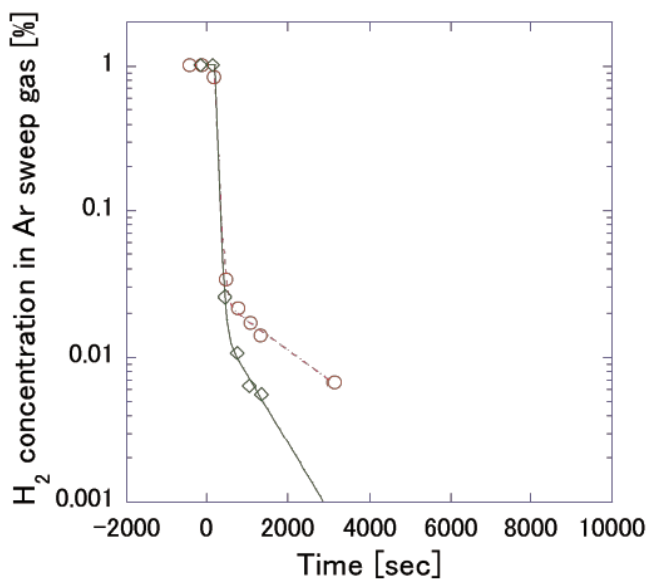

Fig. 4 Hydrogen release behavior of FLiNaK and Ti-containing FLiNaK at 903 K. Diamond and solid line: pure FLiNaK. Circle and dashed line: Ti-mixed FLiNaK. 


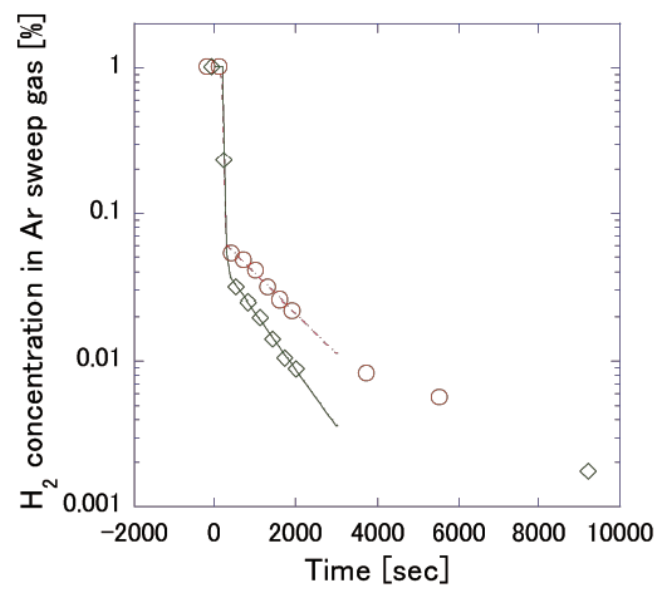

Fig. 5 Hydrogen release behavior of FLiNaK and Ti-containing FLiNaK at 973 K. Diamond and solid line: pure FLiNaK. Circle and dashed line: Ti-mixed FLiNaK.

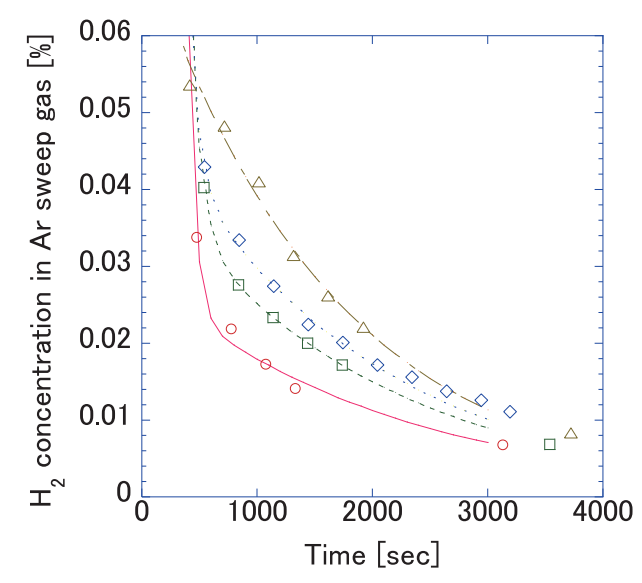

Fig. 6 Released hydrogen from Ti-containing FLiNaK. Circle and solid line: $798 \mathrm{~K}$, Square and dashed line: $853 \mathrm{~K}$, Diamond and dotted line: $903 \mathrm{~K}$, Triangle and chain line: $973 \mathrm{~K}$.

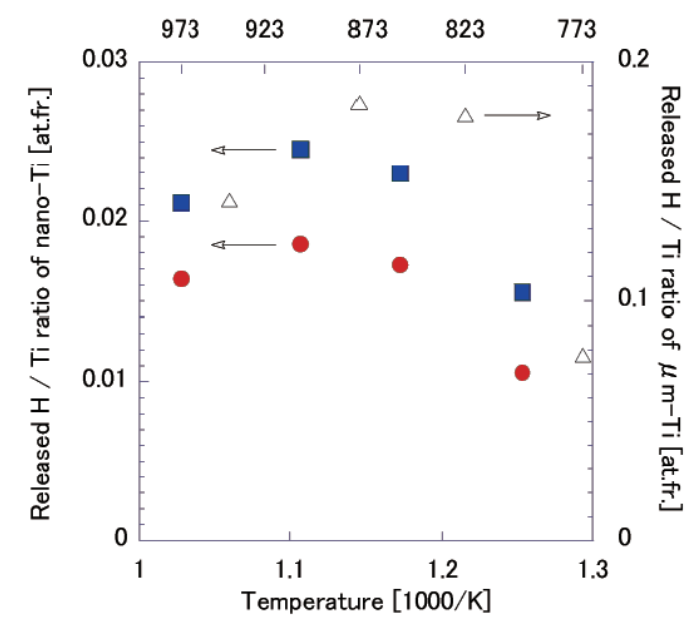

Fig. 7 Released hydrogen from the Ti powder. Circle: nano-Ti from $500 \mathrm{sec}$ to $3000 \mathrm{sec}$. Square: nano-Ti from $500 \mathrm{sec}$ to infinite time. Triangle: $\mu \mathrm{m}-\mathrm{Ti}$ charged by $5-\mathrm{kPa} \mathrm{H}_{2}$ gas [2].

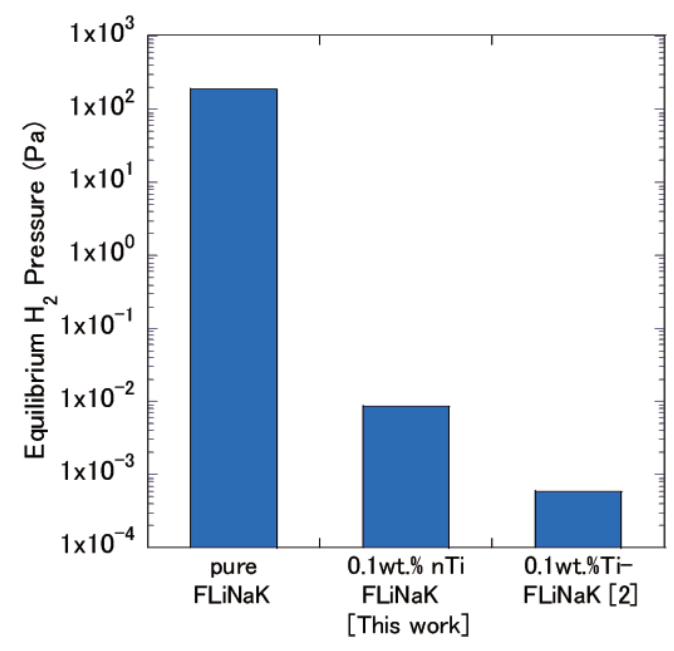

Fig. 8 Hydrogen equilibrium pressure for $1 \mathrm{wppb}-\mathrm{H}$ in the FLi$\mathrm{NaK}$ mixture at $973 \mathrm{~K}$.

present study.

The result at $903 \mathrm{~K}$ showed the highest hydrogen release even though the hydrogen solubility of titanium ideally becomes lower at higher temperatures [4]; this result can be attributed to the effect of surface reactions. Almost the same tendency was observed in our previous research [2], in which $\mu \mathrm{m}$-sized titanium powder was used. The hydrogen release $(\mathrm{H} / \mathrm{Ti}$ ratio) is almost $1 / 10$ of the values reported in previous studies. Assuming that the hydrogen solubility of Ti obeys the Sievert's law, i.e., the hydrogen concentration in a metal is linear to the square root of the hydrogen partial pressure, from the result obtained at $973 \mathrm{~K}$, hydrogen equilibrium pressure for $1 \mathrm{wppb}$ was calculated and is shown in Fig. 8. This result indicates that using nano-Ti powder shows no obvious improvement in hydrogen solubility control.

\section{Summary}

Hydrogen release behavior of the molten salt FLiNaK containing $0.1 \mathrm{wt} . \%$ nano-Ti powder was investigated. For Ti-containing FLiNaK, faster hydrogen release was observed at higher temperatures and the volume of released hydrogen was significantly higher than that from pure FLi$\mathrm{NaK}$. This indicates the higher hydrogen solubility of the molten salt mixture. However, the ratio of released $\mathrm{H} / \mathrm{Ti}$ was smaller than that obtained with $\mu \mathrm{m}$-Ti powder.

\section{Acknowledgments}

This work was supported by a JSPS KAKENHI Grant-in-Aid for Scientific Research (A) 25249133 and NIFS budget code UFFF021-2.

[1] A. Sagara et al., Fusion Eng. Des. 89, 2114 (2014).

[2] J. Yagi et al., Fusion Eng. Des. 98, 1907 (2015).

[3] Y. Ito et al., Molten Salts Chemistry: From Lab to Applications (Elsevier, 2013) p.269.

[4] A. San-Martin and F.D. Manchester, Bull. Alloy Phase Diagrams 8, 30 (1987). 